\title{
Day-ahead industrial load forecasting for electric RTG cranes
}

\author{
Feras ALASALI $^{1}{ }^{\circledR}$, Stephen HABEN ${ }^{2}$, Victor BECERRA ${ }^{3}$, \\ William HOLDERBAUM ${ }^{1,4}$
}

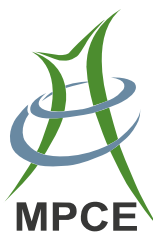

\begin{abstract}
Given the increase in international trading and the significant energy and environmental challenges in ports around the world, there is a need for a greater understanding of the energy demand behaviour at ports. The move towards electrified rubber-tyred gantry (RTG) cranes is expected to reduce gas emissions and increase energy savings compared to diesel RTG cranes but it will increase electrical energy demand. Electrical load forecasting is a key tool for understanding the energy demand which is usually applied to data with strong regularities and seasonal patterns. However, the highly volatile and stochastic behaviour of the RTG crane demand creates a substantial prediction challenge. This paper is one of the first extensive investigations into short term load forecasts
\end{abstract}

CrossCheck date: 9 January 2018

Received: 12 September 2017 / Accepted: 9 January 2018/Published online: 27 February 2018

(C) The Author(s) 2018. This article is an open access publication

$\bowtie$ Feras ALASALI

F.M.M.Alasali@student.reading.ac.uk

Stephen HABEN

Stephen.Haben@maths.ox.ac.uk

Victor BECERRA

victor.becerra@port.ac.uk

William HOLDERBAUM

w.holderbaum@ @reading.ac.uk

1 School of Systems Engineering, University of Reading, Berkshire, UK

2 Mathematical Institute, University of Oxford, Oxford, UK

3 School of Engineering, University of Portsmouth, Portsmouth, UK

4 School of Engineering, Metropolitan Manchester University, Manchester, UK for electrified RTG crane demand. Options for model inputs are investigated depending on extensive data and correlation analysis. The effect of estimation accuracy of exogenous variables on the forecast accuracy is investigated as well. The models are tested on two different RTG crane data sets that were collected from the Port of Felixstowe in the UK. The results reveal the effectiveness of the forecast models when the estimation of the number of crane moves and container gross weight are accurate.

Keywords Rubber-tyred gantry (RTG) cranes, Correlation analysis, Exogenous variables estimation, Artificial neural networks, Time series forecast modelling

\section{Introduction}

Over the last decade, the amount of international trading worldwide has increased rapidly and ports are facing significant environmental and energy challenges such as rising fossil fuels prices and greenhouse emissions. Diesel rubber-tyred gantry (RTG) cranes are usually environmentally and economically inefficient due to their use of more fuel, fuel cost volatility and generation of gas emissions such as carbon dioxide and nitrogen oxides. One way to reduce carbon gas emissions and operation costs is to convert from diesel to electrified RTG cranes [1,2]. Consequently, the electrical power consumption at port substations has been increasing as a result of a rise in the number of electrified RTG cranes which connected to low and medium voltage networks. The use of electrified RTG cranes can reduce maintenance and repair parts costs by around 30\% and green gas emissions by between $25 \%$ and $70 \%$ compared to diesel RTG cranes [1, 3]. However, shifting from diesel to electrified RTG cranes will lead to an increase in peak 
demands and power consumption at the port substations. In this situation, ports may need to upgrade the electrical infrastructure to meet this rise in demand [3]. In addition, there is a gap and lack of understanding of the ports and the RTG crane energy demand behaviour. This understanding is vital for developing power generation strategies to reduce the environmental effects of gas emissions and peak demand problems [4]. Load forecasting is an important tool for the estimation of any financial or technical risk that may occur in the future resulting from demand inconsistency. An accurate forecast is an effective solution for energy management system problems such as load shedding, peak demand and electrical infrastructure development [5]. In power system applications, short-term load forecasting has been used widely for operation scheduling, power system stability and economic operation [6, 7]. A large variety of methodologies and models have been employed in order to achieve an accurate short-term load forecast. These models are mainly divided into three categories:

1) Traditional or statistical methods: for example, autoregressive integrated moving average with exogenous variable (ARIMAX) and autoregressive with exogenous variable (ARX) [8].

2) Artificial intelligence methods: such as artificial neural networks (ANN) [9] and support vector machine (SVM)[10].

3) Hybrid forecast system: for instance, ARMAX-SVM [11] and regime switching models [12].

Forecast models play a vital role in many electrical power system applications, such as planning, operation and energy markets. ARIMAX and ANN forecasting concepts have been applied widely in different energy applications such as buildings, industrial loads and renewable energy $[13,14]$. It should also be beneficial to apply these techniques to forecasting the RTG crane demand in order to improve the understanding of load behaviour which can help to reduce peak demand and gas emissions. There are many publications that have discussed and developed load forecasting models in the literature, which successfully predict the highly volatile targets such as wind speed [15], solar radiation [16], microgrid systems and building demand by using ANN and ARIMAX techniques. However, these systems in the literature have clear physically explanatory relationships with exogenous variables compared to the RTG crane demand. For example, [17] developed two different ANN models for high and low frequency data sets with seasonal data points which is captured by using Wavelet decomposition in order to forecast a 24-hour ahead peak demand for Irans national grid. In addition, time series methods are widely used to predict electricity prices. Reference [18] proposed an
ARIMAX model to forecast the electrical power prices at Nord Pool, the model included exogenous variables such as temperature, wind speed and reservoir level which mainly reflect seasonal trends in the weekly spot price and it helped to reduce the forecast error.

It should be noted that the forecast models presented in the literature for buildings, industrial loads and smart grids have used the seasonality correlation and the exogenous variables that have a clear relationship with forecast target to predict the load demand. Unlike previous studies, the electrified RTG crane demands are: (1) highly volatile and stochastic; (2) not include a clear seasonality or patterns, can help to improve the forecast quality; (3) highly unpredictable behaviour due to the effect of human (crane driver) on the crane moves and loads.

Furthermore, to the authors knowledge, while only a single study discussed the RTG crane forecasting problem [4], there are no studies found which specifically consider forecasting of load for electrified RTG crane by estimating the number of moves and container gross weight and examine different input variables. Reference [4] used ARIMAX and ANN models with one hidden layer to predict a 24-hour RTG crane demand. However, they assumed that the exogenous variables are known in advance without examining the impact of inputs error on the forecast accuracy. In addition, the RTG crane forecast models do not investigate the effect of exogenous input variables on the forecast performance [4].

Aiming to fill the gap in the literature and address the lack of understanding of the energy demand behaviour at port applications, this paper attempts to develop short term forecast models to predict the electrified RTG crane load one-day ahead. The forecast models in this paper (ANN with two hidden layers, ARIMAX, ARX, ARIMA, AR) are used with estimation technique to select the number of cane moves and container gross weight and examine the impact of inputs variables error on the forecast models. In this paper, the forecast models are tested using data collected from two RTG cranes over three separate time periods. The key contributions of this paper are summarized as follows:

1) This paper uses two new forecast models that show more accurate results compared to the literature. Firstly, an ANN system with two hidden layers that help to improve the forecast model performance. Secondly, an ARX model that has a similar performance to the ARIMAX model but with the advantage of having a higher speed to calculate the AR coefficient.

2) This work examines the impact of the accuracy of the exogenous variables on the forecast model performance in order to develop an accurate forecast model. 
3) An approach is proposed for estimation of the exogenous variables (number of crane moves and container gross weight). This estimation helps to check the forecast model performance for a range of errors at the forecast model inputs (exogenous variables).

4) In this work, the forecast models have been tested over three different time periods. In addition, all forecast models have been trained using one RTG crane testing data set and tested with two different RTG crane data sets.

The remaining sections of this article are structured as follows. Section 2 introduces the methodology of the proposed models. Section 3 discusses the proposed models approach in detail. In Section 4, the forecast models results are given and discussed. Finally, the conclusions are presented in Section 5.

\section{Methodology}

Electrified RTG cranes are used in the intermodal operations for container handling at ports. The RTG demand behaviour is mainly non-smooth, volatile and without obvious patterns or seasonality in the demand time series which increases the challenge of forecast the crane load. In this research, ANN and ARIMAX models are developed to predict the hourly electrified RTG crane demand at hour $t+n$ where $t$ is the hour time and $n=1,2, \ldots, 24$. Furthermore, we examine the impact of some exogenous variables $\left(X_{t}, Y_{t}\right)$ on the forecast models performance. A general schematic of the load forecasting procedure is shown in Fig. 1. This section presents the methodologies we implement for electrified RTG crane load forecasting.

\subsection{ANN}

ANN are mathematical models constructed by multiple layers of artificial neurons and they are interconnected by synaptic weight $W_{i n}$ from each individual neuron $X_{n}$ in one layer to each neuron in the next layer [10].

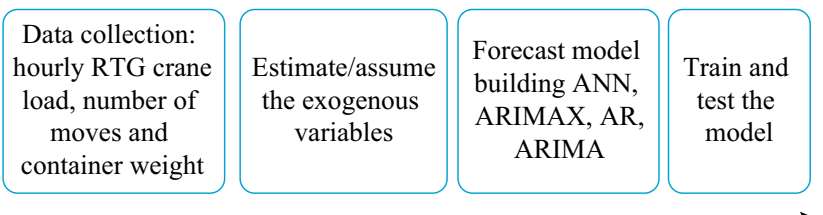

Fig. 1 General schematic of load forecasting procedure implemented in this paper $y_{i}=\sum_{n=1}^{m} W_{i n} X_{n}$

where $y_{i}$ is the summation of synaptic weight $W_{i n}$ (between the input neuron $n$ and the hidden neuron $i$ ) multiplied by the outputs of each individual neuron $X_{n}$ and $m$ is the number of neurons. In the structure of a typical individual artificial neuron, the summation of input signals $y_{i}$ from several synapses is transferred through an activation function $F$ described by (2) for the chosen function sigmoid activation.

$F\left(y_{i}\right)=\frac{1}{1+\mathrm{e}^{-y_{i}}}$

The ANN is trained to modify the weights by calculating the error of the $i$ th neuron $\left(\gamma_{i}\right)$ throughout the hidden layer presented by (3) and (4).

$\gamma_{i}=F\left(y_{i}\right)\left(\widehat{a}_{n}-a_{n}\right)$

where $\widehat{a}_{n}$ and $a_{n}$ are the predicted value and the actual target value of $n$th neuron in the output layer, respectively. In (4), the error information $\gamma_{i}$ is used to update the synaptic weight $W_{\text {in }}$ :

$\Delta W_{i n}=\omega \gamma_{i} O_{i}$

where $\omega$ is the training rate and $O_{i}$ is the output of the $i$ th neuron. Next, the weight correction $\Delta W_{\text {in }}$ is used to modify the old synaptic weigh $W_{i n, o l d}$ :

$W_{\text {in, new }}=\Delta W_{\text {in }}+W_{\text {in,old }}$

where $W_{\text {innnew }}$ is the updated weight between the neuron $n$ and the hidden neuron $i$. Finally, after the ANN model is trained, the model can be generalised and tested on similar sets.

\subsection{ARIMAX}

The ARIMA technique is a time or statistical series method that formulates the historical data as a function of time to predict the future value $a_{t}$. An $\operatorname{ARIMAX}(p, d, q)$ model for a time series is described by (6) and (7) [19].

$$
\begin{aligned}
& a_{t}^{(d)}=C+\sum_{i=0}^{h} \mu_{i} X_{t-i}+\sum_{i=1}^{p} \psi_{i} a_{t-i}^{(d)}+\sum_{i=1}^{q} \varphi_{i} \epsilon_{t-i} \\
& a_{t}^{(d)}=a_{t}^{(d-1)}-a_{t-1}^{(d-1)}
\end{aligned}
$$

where $a_{t}^{(d)}$ is the differenced series at time $t$ (defined by (7) with $\left.a_{t}^{(0)}=a_{t}\right) ; \sum_{i=0}^{h} \mu_{i} X_{t-i}$ is the $X$ variables term; $\sum_{i=1}^{p} \psi_{i} a_{t-i}^{(d)}=\operatorname{AR}(p)$ is the autoregressive term of order $p$; 
$\sum_{i=1}^{q} \varphi_{i} \epsilon_{t-i}=\operatorname{MA}(q)$ is the moving average term of order $q$; $\psi_{i}$ is the coefficient of $\operatorname{AR}(p)$ term; $\varphi_{i}$ is the coefficient of the $\operatorname{MA}(q)$ term; $\epsilon_{t}$ is error term; $\mu_{i}$ is the coefficient parameter for the exogenous variable $X_{t-i}$ for the number of exogenous variable $h$; $C$ is a constant term. Furthermore, ARX, ARIMA and AR are subclasses of ARIMAX models. For example, ARX is an integrate of the autoregressive terms $\operatorname{AR}(p)$ with exogenous variables [19].

\subsection{Exogenous variables estimation}

Estimation techniques based on different distribution methods have been used widely for estimating the exogenous variables of forecast models $[5,20]$. In this research, the exogenous variables at hour $t\left(X_{t}\right)$ are the hourly container gross weight $Z_{t}$ and number of crane moves $Y_{t}$ due to the high correlation between these variables and RTG crane demand (at hour $t$ ). However, the exogenous variables for $t+n(n=1,2, \ldots, 24)$ are typically unknown. Aiming to improve the forecast model and examine the impact of the exogenous variables, here we develop models for: (1) estimating both exogenous variables $Z_{t}$ and $Y_{t}$; (2) estimating only one exogenous variable for example $Z_{t}$ and assume that the second variable $Y_{t}$ is known in advance.

We compare these estimation methods to the case when we assume knowing the exact values of the exogenous variable $X_{t}$ in advance. We will estimate the variables by means of random sampling from appropriate distribution functions. As we will show, the exogenous variables are highly correlated hence when one variable is known we must use the conditional probability for sampling.

In order to estimate both exogenous variables, the joint probability distribution has been used in this paper. Since the weight $Z_{t}$ is a continuous variable and the number of crane moves $Y_{t}$ is discrete value, the mixed case of the joint distribution used as:

$f\left(Z_{t}, Y_{t}\right)=f\left(Z_{t} \mid Y_{t}\right) P\left(Z_{t}\right)=P\left(Z_{t} \mid Y_{t}\right) f\left(Z_{t}\right)$

where $f\left(Z_{t}, Y_{t}\right)$ is the probability density function of $\left(Z_{t} \mid Y_{t}\right)$; $f\left(Z_{t} \mid Y_{t}\right)$ is the probability density function of $Z_{t}$ with given $Y_{t} ; P\left(Z_{t}\right)$ is the probability of $Z_{t} ; P\left(Z_{t} \mid Y_{t}\right)$ is the the conditional probability distributions of $Z_{t}$ with given $Y_{t}$ with respect to the marginal distributions $f\left(Z_{t}\right)$. On the other hand, in the case of one of the variables being known (for example $Y_{t}$ ), the conditional probability used to estimate the other exogenous variable $\left(Z_{t}\right)$ is described by:

$P\left(Z_{t} \mid Y_{t}\right)=\frac{P\left(Z_{t} \cap Y_{t}\right)}{P\left(Y_{t}\right)}$

where $P\left(Y_{t}\right)$ is the the probability of $Y_{t} ; P\left(Z_{t} \cap Y_{t}\right)$ is the probability of the joint of $Z_{t}$ and $Y_{t}$. To estimate the joint and conditional distributions and sampling the exogenous variables $\left(Z_{t}, Y_{t}\right)$, the empirical distribution used in this paper is described by:

$F_{n}(X)=\frac{1}{M} \sum_{t=1}^{n} f_{X_{t}, X}$

where $f_{X_{t}, X}$ is an indicator function (one if $X_{t} \leq X$ and zero otherwise); $M$ is the sample size.

\subsection{Load forecasting model evaluation}

To assess the performance of a forecasting model or compare different forecasting techniques for a specific time series or a particular application, it is important to define the performance evaluation method. The forecast accuracy or forecast error can be measured by using different techniques [21]. Reference [21] shows that there are four main performance evaluation techniques that have been used to evaluate the accuracy of load forecasting models. In this paper, the mean absolute percentage error (MAPE), root mean square error (RMSE) and mean absolute error (MAE) have been used to evaluate the models performance. However, MAPE is one of most common load forecasting evaluation methods. This technique uses the percentage terms to make it easy to interpret [21].

$$
\begin{aligned}
& \text { MAPE }=\frac{100}{N} \sum_{i=1}^{N}\left|\frac{a_{i}-\widehat{a}_{i}}{a_{i}}\right| \\
& R M S E=\sqrt{\frac{\sum_{i=1}^{N}\left(a_{i}-\widehat{a}_{i}\right)^{2}}{N}} \\
& M A E=\frac{\sum_{i=1}^{N}\left|a_{i}-\widehat{a}_{i}\right|}{N}
\end{aligned}
$$

where $a_{i}$ is the load of an electrified RTG crane; $\widehat{a}_{i}$ is the forecasted load; $i$ is the time step; $N$ is the number of observations.

\section{Data analysis and forecasting models}

The power demand of an electrified RTG crane is very difficult to predict due to the nature of the crane operator's actions. In addition, the factors which are normally used for power distribution forecasting such as temperature and seasonality variables do not pertain to the crane demand. Electrified RTG crane loads exhibit a volatile behaviour (see Fig. 2). To achieve a satisfactory load forecast for RTG crane loads, the forecast model must be able to capture the correlation between the load in the time series, 


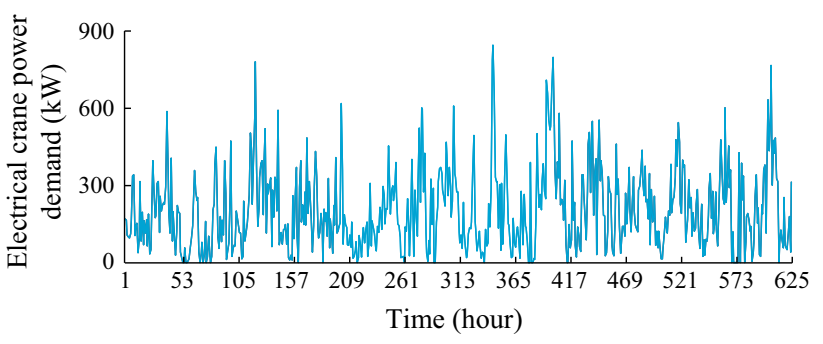

Fig. 2 Hourly three-phase electrified RTG crane power demand from 15th of April to 10th of May in 2016

the number of crane moves and container gross weight, as these factors are most effective on forecast model accuracy [4]. As seen in Fig. 2, the exogenous variables are unknown in advance and they show highly volatile and unpredictable behaviour. Ports worldwide are converting from diesel to electrified RTG cranes with fully automated work solutions to achieve the gas emissions and safety of life at sea convention (SOLAS) requirements. From 1st of July 2016 all shippers, freight forwarders and ports around the world are required to follow the new SOLAS requirements. This requires the gross weight of each container to be recorded when leaving and arriving at any port $[22,23]$. These new international requirements and rules will help ports around the world know the exact weight of the container in advance. Furthermore, this makes the assumption of knowing the container gross weight and number of crane moves in advance achievable and realistic in the future especially with fully automated ports. To test the effect of estimating the container weight and number of moves on the accuracy of the forecast we have developed different forecast models to predict RTG crane loads 24 hours ahead using variations on the input variables. In total we test four variations on how we use the exogenous variables which are as described below:

1) Estimating the exogenous variables (number of crane moves and container gross weight) as described in Section 2.3.

2) Estimating one of the exogenous variables and assuming the other variable is known in advance.

3) Assuming both exogenous variables are known in advance.

4) Generating the forecast models without the exogenous variables.

\subsection{Data collection and analysis}

The measured data were collected at the Port of Felixstowe in the UK from two electrified RTG cranes over three different periods during normal operation days. The first data set was collected from 15th of April to 10th of
May, 2016 and the second data set from 7th of September to 10th of October, 2016 with both sets coming form the same crane. The third data set was collected from another RTG crane over seven days from the 7th to the 13th of December, 2016. The first data set is divided into 21 days of training data and five days of testing data. The second and third data sets are used as testing data sets. Due to the non-smooth behaviour of the RTG crane load and the lack of seasonality or trends over the time series (as described in the following section) and motivated by the literature $[2,4]$, we assume that the gap in the time series will have negligible effect on the forecast results. In this paper, the testing period with 46 days data set allows us to evaluate the forecast model over three different time periods and test the transferability of the model trained on one crane to other cranes with the same specifications.

We analyse the data to investigate different patterns in the RTG crane demand series. The breakdown of the crane demand by hour of the day in Fig. 3 shows that the demand has stochastic, non-smooth and volatile behaviour and the hourly patterns are not obvious. In addition, the $R^{2}$ for a linear model is calculated to find the correlation strength between the current and historical demand for 1600 observations. The calculation results exhibit a low $R^{2}$ value of under 0.29. In other words the linear model only explains $29 \%$ of the load variability. To find any correlation or patterns in the time series, the partial autocorrelation function (PACF) is calculated over 500 time lags, this is shown in in Fig. 4. The analysis of PACF with large numbers of lags can find trends and seasonalities in the data set in case the significant lags are repeated with specific pattern. The significant lags in the PACF show no clear pattern or seasonalities. This indicates that an autoregressive model may have limited ability to forecast accurately. However, the significant lags between lag 450 and lag 500, as shown in Fig. 4 are randomly distributed without a main large spike that decreases after a few lags or follow by a damped wave which can present a moving average term or autocorrelation pattern. In addition, they are likely an artifact from highly variable time series and this is referred

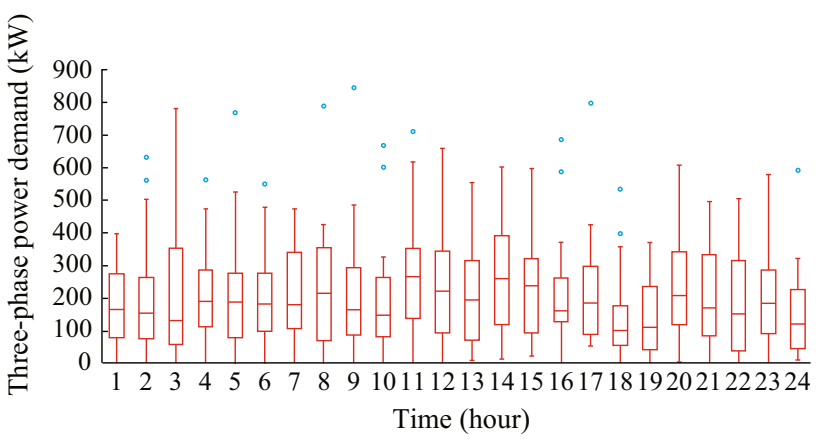

Fig. 3 Breakdown of electrified RTG crane demand by hour of a day 


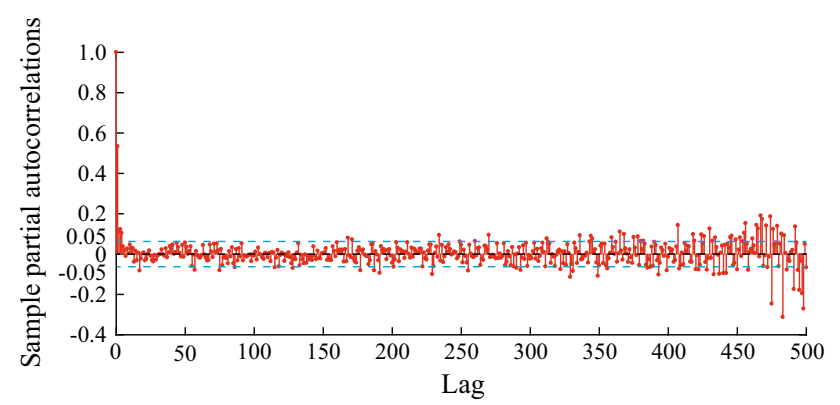

Fig. 4 PACF plot for an electrified RTG crane for 500 time lags

by the lack of regular patterns. The distribution of these significant lags gives a significant sign that the training data set is highly volatile and stationary.

Due to the highly volatile and unpredictable behaviour of the electrified RTG crane demand and the difficulties of finding seasonality trends, the correlation analysis is now extended to consider the exogenous variables. The collected data show a high correlation between the power demand of the electrified RTG cranes number of crane moves and container gross weight with an $R^{2}$ value for a linear model equal to 0.89 , as seen in Fig. 5. Both variables (number of crane moves and container gross weight) are important for developing an accurate forecast model. In this study, the exogenous variables are applied to the forecast model as described above. The correlation between the RTG crane power demand, number of crane moves and container gross weight is significant with an $R^{2}$ value but the effect of human unpredictability on the demand is still present and can be seen in Table 1. The path for the crane move is decided by the crane drivers as they may choose to move the container through arc, square or oscillatory paths which means variations in the amount of energy used for the same number of moves and container weight. Table 1 shows that the historical electrified RTG crane data include different power demand values for the same number of moves and container gross weight. For example, on the 12th of September, 2016 at each of the

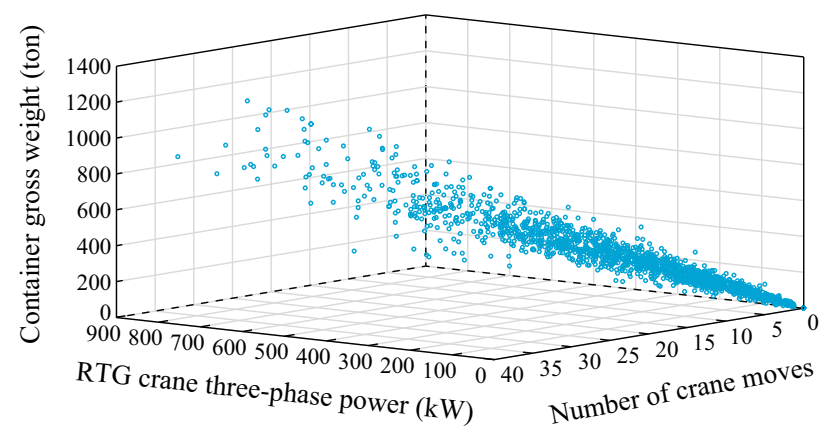

Fig. 5 3D scatter plot for RTG crane demand, container gross weight and number of moves
Table 1 Difference between the power demand values for the same number of moves and container gross weight

\begin{tabular}{llll}
\hline Moves & Weight (ton) & Time in 2016 & Demand (kW) \\
\hline 2 & 46 & 9th of October (11:00) & 37 \\
2 & 46 & 13th of October (09:00) & 23 \\
2 & 59 & 12th of September (06:00) & 28 \\
2 & 59 & 12th of September (09:00) & 47 \\
2 & 59 & 12th of September (14:00) & 48 \\
7 & 184 & 1st of September (15:00) & 86 \\
7 & 184 & 2nd of September (07:00) & 164 \\
7 & 184 & 27th of September (20:00) & 137 \\
\hline
\end{tabular}

following time, 6 a.m., 9 a.m. and 2 p.m., the electrified RTG crane needed two moves per hour to move 59 tons. The human factor is very difficult to predict and this leads to less predictable RTG crane load behaviour. In addition, Fig. 5 shows that larger uncertainty is associated to larger container weights and larger number of moves which increases the difficulties of forecasting the RTG crane demand.

\subsection{Exogenous variables estimation model}

The highly volatile behaviour of the RTG crane demand for the same number of moves and container weight as seen in Table 1 and Fig. 5 increases the difficulty of achieving an accurate forecast model. In this research, the estimation of the exogenous variables is presented to examine the wide variety of the inputs and outputs. The exogenous variables of the forecast models (container gross weight and number of crane moves) are obtained using the empirical distribution with a joint probability model to estimate both the exogenous variables $\left(Z_{t}, Y_{t}\right)$ and conditional probability to estimate one of the variables in condition the other variable is given for $t+n$ $(n=1,2, \ldots, 24)$, as described in Section 2.3. For estimating both exogenous variables through the joint probability with empirical distribution, the 2D histogram of the exogenous variables data sets is displayed in Fig. 6 with 100 bins. Each of the histogram bins (bars) presents the joint probability distribution for the exogenous variables (container gross weight and number of crane moves) and increases the numbers of bins help to reduce the number of choices for the exogenous variables. Next, the empirical distribution is used to sample the variables $\left(Z_{t}, Y_{t}\right)$ from the joint probability distribution by using (8) and (10) in Section 2.3. On the other hand, if one of the exogenous variables is known, the conditional distribution is used to determine the other variable by using the histogram defined for the known variable. From Fig. 7 if the number of 


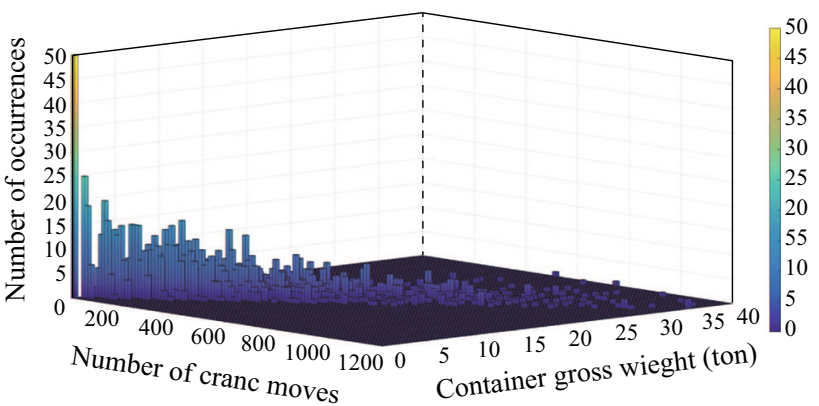

Fig. 6 Illustration of number of crane moves and container gross weight data in a $2 \mathrm{D}$ histogram distribution

moves $Y_{t}$ is 2 , the conditional distribution is defined by the dots only which inline with $Y_{t}=2$. This defines a new histogram for the number of moves $\left(Y_{t}=2\right)$ with the container gross weight $Z_{t}$ and then we sample the $Z_{t}$ value from it by using the empirical distribution function as described by (9) and (10) in Section 2.3.

\subsection{ANN}

To forecast the hourly power demand of an electrified RTG crane for the next 24 hours, two ANN models are used, as seen in Fig. 8. The proposed models are feedforward ANN activated by a sigmoid function and trained by the Levenberg-Marquard algorithm that has been widely used with highly volatile forecast targets. Generally, there is no specific or favourite method that can guarantee the best number of neurons for each layer and the number of layers [10]. However, an increase in the number of neurons and layers may generate over fitting and overestimation problems and increase the training time required [10]. An experiment was carried out based on the number of hidden layer from 1 to 5 and number of hidden neurons $5,10,15$, 20 and 25 in this study to select the best parameters for the forecast model that produces the minimum forecast error. In general, one or two hidden layers with a sufficient number of neurons is able to handle a wide range of forecasting problems without leading to over-fitting [24]. The ANN forecast model with two hidden layers generates the minimum forecast error for the same number of hidden

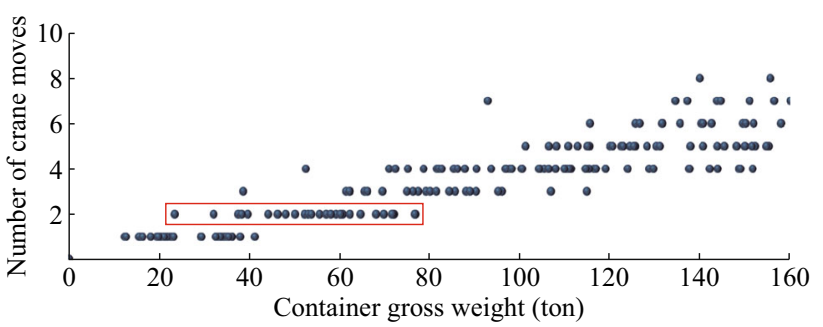

Fig. 7 Illustration of exogenous variables correlation for conditional probability when $Y_{t}$ is 2

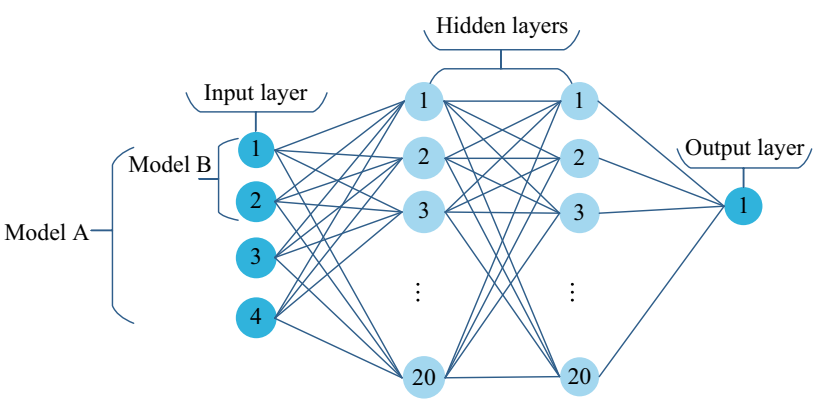

Fig. 8 Representation of ANN forecast models: Model A employs four input neurons while Model B uses two input neurons

neurons compared to the [4] with single hidden layer and ANN models with 3 to 5 hidden layers over the same data set.

\subsubsection{Model A}

The objective of Model A is to investigate the performance of a forecast model that does not include the exogenous variables. The RTG crane demand data analysis in Section 3.1 shows that the autocorrelation over the time series is weak. However, for this model we choose the lags with the highest correlation values. These values are used as input neurons for Model A as follows: (1) the average of the previous day load; (2) the average of the previous week load; (3) the same hour load for previous day; (4) the previous hour load.

In this study, the parameters of Model A are: (1) 4 input neurons; (2) 2 hidden layers; (3) 20 neurons in each hidden layer; (4) 1 output layer referring to the RTG crane demand of one hour in order to simplify the model network. This load forecasting is repeated 24 times for each hour of the next day to forecast the next day demand. In this paper, we use Model A as a benchmark to compare against the other forecast models.

\subsubsection{Model B}

Model B is structured to generate the forecast models using the exogenous variables. This model is a variation of the benchmark model. Which aims to build an RTG crane demand forecast model using the following exogenous variables: (1) the number of crane moves; (2) container gross weight.

The exogenous variables have been selected based on correlation analysis in Section 3.1. Model B is divided into four models based on the exogenous variables are known or estimated:

1) Model B1: assuming both exogenous variables are known. 
2) Model B2: estimating the number of crane moves while assuming the container gross weight is known.

3) Model B3: estimating the container gross weight while assuming the number of crane moves is known.

4) Model B4: estimating both of the exogenous variables.

Model B is aims to utilise the correlation between exogenous inputs and RTG crane demand to improve the ANN forecast performance. Throughout this paper, we refer to the number of crane moves and container gross weight as the exogenous variables.

\subsection{ARIMAX and ARX}

This section presents ARIMAX, ARX, ARIMA and AR models to forecast the power demand of an electrified RTG crane. The ARX model is an extension of the $\operatorname{AR}(p)$ model, the autoregressive term, with exogenous variables, as shown in Fig. 9. The ARIMAX modelling procedure is presented in Section 2.2 and described by (6) and (7). After the data collection stage, we first confirmed that the time series is stationary using the augmented Dickey-Fuller (ADF) test. To identify and choose the best orders of the ARIMAX parameters $(p, d, q)$, we consider the ACF and PACF plots for the crane demand in Fig. 4.

The ACF and PACF plots show a number of positive and significant lags but do not show obvious signs of repetition or seasonalities. Based on the ACF and PACF plots we test a range of $p$ and $q$ parameters using the Bayesian information parameters. The literature review $[13,21]$ shows that in many applications the model parameters are often between 1 to 2 for $p$ and $q$ and between 0 and 2 for $d$. In this paper, the optimal $p, d, q$ are those which give the smallest BIC value. The BIC matrix calculations show that the best ARIMAX model order are equal to $(1,0,2)$. ARIMAX takes into account exogenous variables (the number of crane moves and container gross weight) as input parameters. In addition, since the differencing is not included in this work we simply refer to the ARMA and ARMAX models.

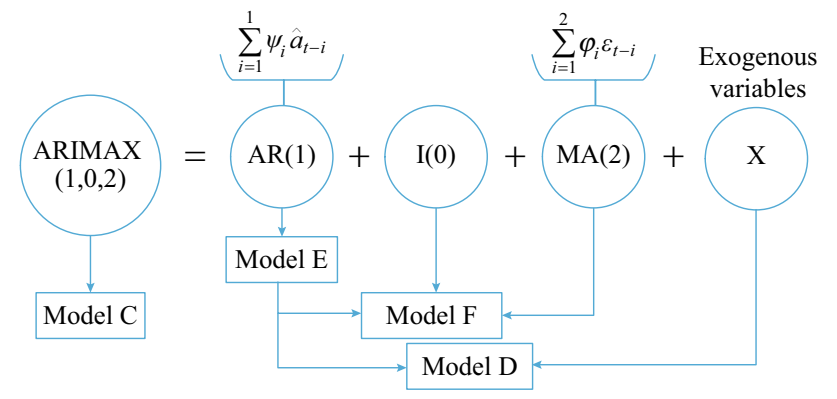

Fig. 9 ARIMAX and ARX forecast models

\subsubsection{Model C}

Model C is the ARIMAX $(1,0,2)$ forecast model. This model is aims to examine the exogenous variables as input parameters similar to Model B. In addtion, the ARIMAX model is divided into four models: Model C1, Model C2, Model C3 and Model C4 analogous to Model B.

\subsubsection{Model D}

Model D (ARX) is a variation and class of ARIMAX. The main advantage of an ARX model is the high speed at calculating the forecast and the model coefficients. The $\operatorname{ARX}(p)$ order based on BIC calculations for the available data sets gives $p=1$. The BIC matrix was calculated and tested for $p$ values from 1 to 24 in order to: (1) cover all significant lags in ACF and PACF plots especially when there is no trend or repeating values for the significant lags as shown in Fig. 4; (2) cover one day lags (24 lags); (3) cover large numbers of lags compared to the literature.

Furthermore, to evaluate the exogenous variables effect on the forecast model, the ARX model is divided into four models similar to Model B and Model C.

\subsubsection{Model E and Model F}

In this study, Model E (AR) and Model F (ARIMA) are forecast models without the exogenous variables. The ARIMA and AR forecast model order parameters are equal to $(1,0,2)$ and (1) respectively based on the BIC calculations. In addition to Model A, Model E and Model F are used as benchmark.

\section{Results and discussion}

A total of 21 days of data from crane number 1 , as described in Section 3.1, are used for training each model separately. In addition, three testing periods are utilised to assess the prediction performance of the proposed forecast models. The testing data set includes 46 days of crane operations with the total number of hours equal to 1104 from two different electrified RTG cranes. The MAPE, RMSE and MAE values are used to measure the model performance, see (11) to (13). In this section, the forecast performance for all models are presented and then the model that performed best is further analysed.

\subsection{Overall comparisons}

Firstly, the MAPE is calculated for each day of the three testing periods and plotted for each model in Fig. 10, in addition, the overall MAPE for each model is presented in 


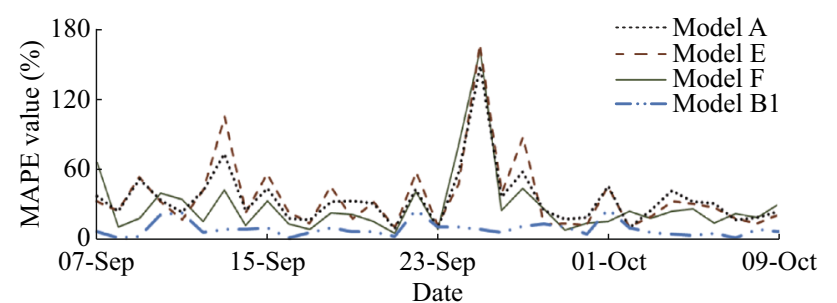

(a)

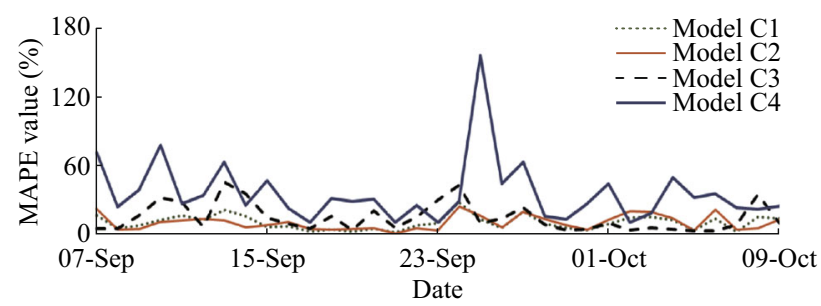

(b)

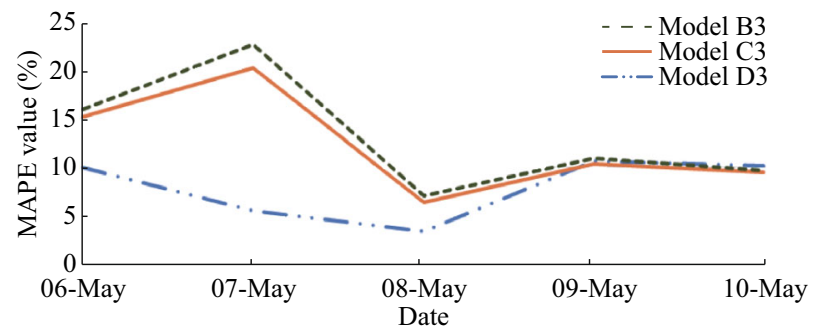

(c)

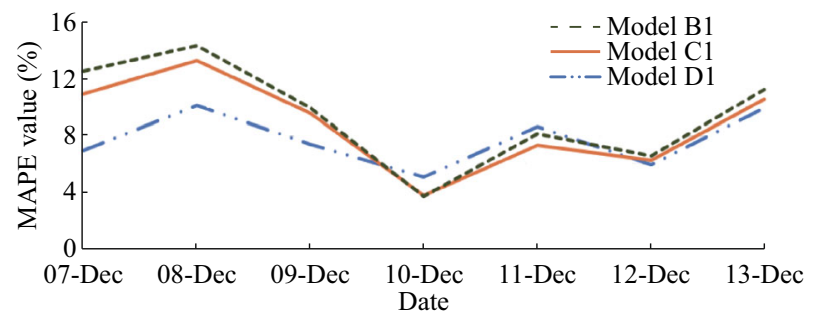

(d)

Fig. 10 Daily MAPE results over three testing periods

Table 2. From Fig. 10 and Table 2, Model B1 provides the highest prediction accuracy over 3 testing periods by considering the overall performance. For all three testing periods, Model A outperforms Model E and Model F. The MAPE for Model A is 23.2\%, 29.2\% and 19.7\% for testing periods 1, 2 and 3, respectively. From Fig. 10a it is seen that by using the exogenous variables in Model B1, the performance clearly improves compared to Models A, E, F that use the RTG crane historical load data only. The MAPE curve of Model B1 in Fig. 10a shows a sign of stability over the testing period compared to benchmark models that exhibit extreme peaks. This is due to the weak daily and weekly trends in the data. Furthermore, the high error peaks in Fig. 10 correspond to low load values and can be explained the significant disturbance that introduced by the human operator and the large uncertainty of the exogenous variables correlation. Then, the RMSE and MAE methods are applied to evaluate the forecast models performance over the crane data set from 7th of September to 10th of October, 2016 (testing period 2). Table 3 shows that the Model B1 outperforms all other models and provides the minimum RMSE and MAE values by $14.2 \mathrm{~kW}$ and $11.6 \mathrm{~kW}$, respectively. Model E generates the highest RMSE and MAE values over the testing period by $57.4 \mathrm{~kW}$ and $46.2 \mathrm{~kW}$.

\subsection{Effect of estimating exogenous variables}

In order to improve the forecast performance and reduce the error peaks, the exogenous variables have been used in this paper. Tables 2 and 3 show that the proposed forecast models that estimate one of the exogenous variables (for example Model C2 ) or know both of them (for example Model D1) give significant improvements over the benchmark models (for example Model E ) by MAPE $26.3 \%$ and $26 \%$, respectively, over the second testing period. Furthermore, the RMSE and MAE values of Model C2 decreased by $40.8 \mathrm{~kW}$ and $33 \mathrm{~kW}$, respectively, compared to Model $\mathrm{E}$ for the same testing period. In addition, Model C2 and Model D1 outperform the best model that estimates both exogenous variables (Model B4) with

Table 2 Overall MAPE over three testing periods

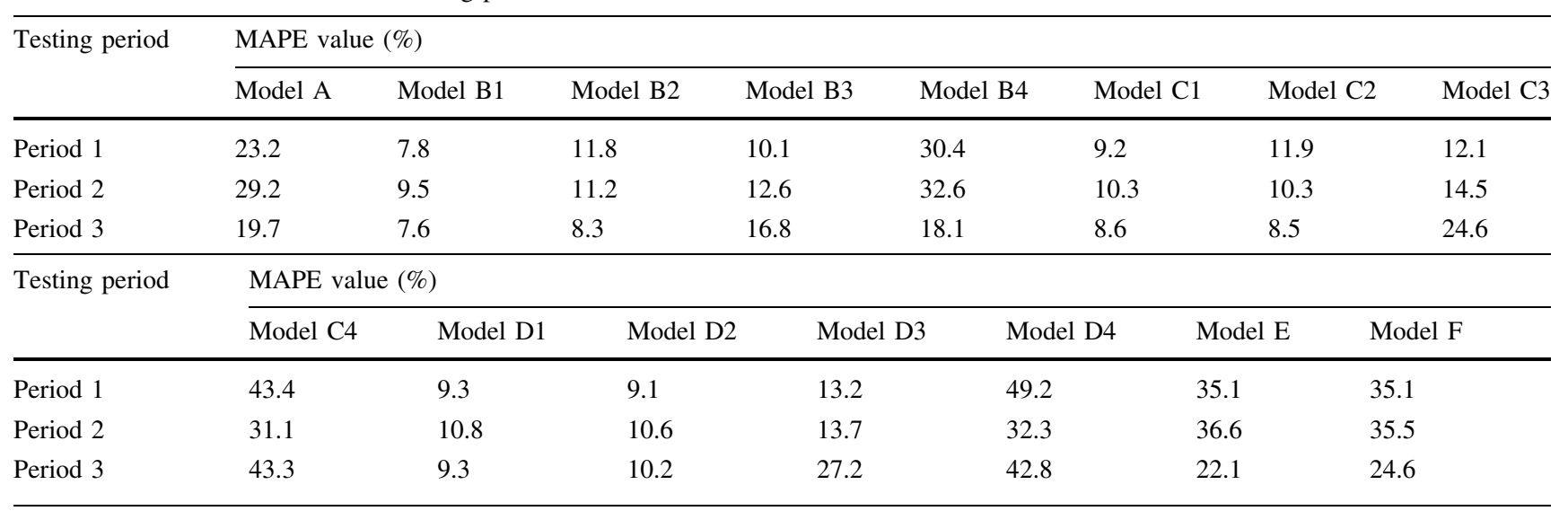


Table 3 Performance of forecast models over 34 day testing period

\begin{tabular}{lll}
\hline Model & MAE value $(\mathrm{kW})$ & RMSE value $(\mathrm{kW})$ \\
\hline Model A & 30.9 & 37.7 \\
Model B1 & 11.6 & 14.2 \\
Model B2 & 14.2 & 17.2 \\
Model B3 & 17.5 & 23.4 \\
Model B4 & 45.1 & 56.5 \\
Model C1 & 12.5 & 15.4 \\
Model C2 & 13.2 & 16.6 \\
Model C3 & 17.9 & 24.8 \\
Model C4 & 43.5 & 54.4 \\
Model D1 & 12.8 & 15.9 \\
Model D2 & 13.6 & 16.8 \\
Model D3 & 18.2 & 25.9 \\
Model D4 & 43.9 & 55.2 \\
Model E & 46.2 & 57.4 \\
Model F & 45.8 & 56.9 \\
\hline
\end{tabular}

$11.9 \%$ and $9.3 \%$ compared $30.4 \%$ over the first data set. Overall, models that estimate both exogenous variables exhibit very large errors. This indicates that, with the current data set, using the historical electrified RTG crane power demand and the estimation of both exogenous variables as inputs is not recommended.

To evaluate the impact of estimating the exogenous variables compared to assuming that the variables are known inputs to the forecast models, Fig. 10b is analysed. The ARIMAX model that estimates the number of crane moves only (Model C2) and Model C1 (the ARIMAX model with the assumption that the exogenous variables are known) perform in a similar way. Furthermore, the MAPE curves of Model C2 and Model C1 show a better performance and stability compared to Model C4 (the ARIMAX model that estimates the container gross weight). This leads to the conclusion that accurate container gross weight has a more significant impact on the forecast performance than number of crane moves. In addition, the daily MAPE results in Fig. 10b shows that the forecast models that estimate either one of the exogenous (Model C2 and Model C3) outperform Model C4, which estimates both variables. Fig. 10b shows that each of the prediction models with an uncertain estimation of the exogenous variables performs differently based on the type of variable.

Fig. 10c presents the daily MAPE results over the first testing period for the ANN, ARIMAX and ARX models that estimate the container gross weight (Model B3, Model C3, Model D3). During the first testing period the ANN model (Model B3) shows a better performance compared to Model C3 and Model D3. However, MAPE results of Models B3, C3 and D3 on 9th and 10th of May are roughly equal. In order to generalise and examine the forecast models, all models in this paper are tested by using a data set collected from another RTG crane (testing period 3), as seen in Fig. 10d and Table 2. The ARIMAX and ARX models with the assumption that both exogenous variables are known (Model C1 and Model D1) performed in similar way over the this testing period. However, the ANN model (Model B1) shows more accurate result especially from 7th to 9th of December but after that all models performed similarly. In Fig. 10d, the lowest MAPE values for Model B1, Model C1 and Model D1 are 5.1\% , 3.8\% and 3.76\%, respectively. Furthermore, the lowest MAPE values for all models In Fig. 10d are met on 10th of December.

Based on the data set, the forecast models should include the number of crane moves and container gross weight as exogenous variables. Estimation of one or both of the variables helps to reduce the high error peaks, outliers and avoids the impact of low or zero load values during ideal and off operation modes. Moreover, the ANN forecast models show that the significant factor is the types of inputs that are used not the number of inputs. In this paper, our main target is to forecast the RTG crane load for a day ahead and compare different structures of the ANN, ARIMAX and ARX models. We believe that it is preferable to use the exogenous variables to decrease the forecast errors.

\subsection{Forecast error analysis}

Table 2 and Fig. 10 present Model B1 as the most suitable and accurate model over different three testing periods. Model B1, Model C1 and Model D1 are further analysed as follows. The histogram of the error percentage for these prediction models over the three testing periods is plotted and fitted with a normal distribution line in Fig. 11. The error percentage values are distributed between the $-60 \%$ and $60 \%$ range. By investigating the histogram plot, it is observed that a high number of instances are clustered

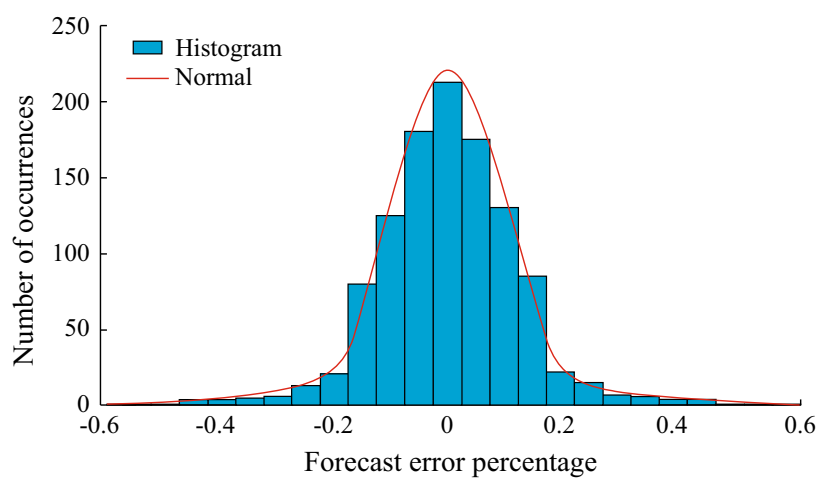

Fig. 11 Illustration of error percentage data in a histogram along with a normal distribution 
around $0 \%$ while many instances are distributed between $-15 \%$ and $15 \%$. In Fig. 11 the normal distribution seems to capture the error percentage. As result, the forecast models (Model B1, Model C1 and Model D1) perform well with normal distribution of around zero error.

\section{Conclusion}

Electrical load forecasting has become a significant evaluation tool for power consumers and producers. The importance of effective and accurate prediction models is to minimise utility risks and power costs and increase competitiveness. While there is a large quantity of load forecasting research, the RTG crane demand forecasting literature is more limited and complex compared to typical distribution loads. There are a number of challenges facing load forecasting of RTG cranes. These challenges can be attributed to three factors. First, there are no clear seasonality trends or pattern over the historical load data. Second, the outliers of the correlation between the exogenous variable and crane demand due to the human factor effect. Third, there is highly stochastic, volatile and nonsmooth load behaviour especially for the low load values. In this research we have implement and tested a number of models to forecast the RTG crane day ahead load. After the RTG crane load series and the exogenous variables are analysed, we examine different options of forecast model inputs. Each model is trained separately using 21 days of data. To verify the prediction ability of the forecast models they are applied to two different cranes over three testing periods. The evaluation methods results of each model with different input variables discussed in this paper show that the ANN model (Model B1) outperforms all other models. Although there are some extreme error peaks, the proposed Model B, Model C and Model D which exclude the estimation of both input variables achieved an acceptable level of prediction accuracy. Our results show that it is not recommended to estimate both exogenous variables in the models. Moreover, the prediction models achieve an acceptable performance when estimating only one exogenous variable with a more significant impact on forecast performance for the accurate container gross weight. This result is very encouraging for ports following the SOLAS requirements and record the container weight before moving them, which will help to forecast and understand the RTG crane demand.

Acknowledgements The authors are grateful to the Port of Felixstowe for providing the electrified RTG cranes data.

Open Access This article is distributed under the terms of the Creative Commons Attribution 4.0 International License (http:// creativecommons.org/licenses/by/4.0/), which permits unrestricted use, distribution, and reproduction in any medium, provided you give appropriate credit to the original author(s) and the source, provide a link to the Creative Commons license, and indicate if changes were made.

\section{References}

[1] Yang YC, Chang WM (2013) Impacts of electric rubber-tired gantries on green port performance. Res Transp Bus Manag 8:67-76

[2] Papaioannou V, Pietrosanti S, Holderbaum W et al (2016) Analysis of energy usage for RTG cranes. Energy 125:337-344

[3] Alasali F, Haben S, Becerra V et al (2017) Optimal energy management and MPC strategies for electrified RTG cranes with energy storage systems. Energies 10(10):1-18

[4] Alasali F, Haben S, Becerra V et al (2016) Analysis of RTG crane load demand and short-term load forecasting. Int $\mathrm{J}$ Comput Commun Instrumen Eng 3(2):448-454

[5] Ziel F, Liu B (2016) Lasso estimation for GEFCom2014 probabilistic electric load forecasting. Int $\mathrm{J}$ Forecast 32(3):1029-1037

[6] Hong T, Fan S (2016) Probabilistic electric load forecasting: a tutorial review. Int J Forecast 323:914-938

[7] Kou P, Gao F (2013) A sparse heteroscedastic model for the probabilistic load forecasting in energy-intensive enterprises. Electr Power Energy Syst 55:144-154

[8] Feuerriegel S, Riedlinger S, Neumann D (2014) Predictive analytics for electricity prices using feed-ins from renewables. In: Proceedings of twenty-second European conference on information systems, Tel Aviv, Israel, 9-11 June 2014, 14 pp

[9] Gui M, Ke D, Gan D et al (2015) Statistical scenarios forecasting method for wind power ramp events using modified neural networks. J Mod Power Syst Clean Energy 3(3):371-380

[10] Kalogirou S (2000) Applications of artificial neural-networks for energy systems. Appl Energy 67(1-2):17-35

[11] Yan X, Chowdhury N (2014) Mid-term electricity market clearing price forecasting utilizing hybrid support vector machine and auto-regressive moving average with external input. Int J Electr Power Energy Syst 63:64-70

[12] Rasmussen L, Bacher P, Madsen H et al (2016) Load forecasting of supermarket refrigeration. Appl Energy 163:32-40

[13] Jiang Y, Chen X, Yu K et al (2017) Short-term wind power forecasting using hybrid method based on enhanced boosting algorithm. J Mod Power Syst Clean Energy 5(1):126-133

[14] Hu W, Min Y, Zhou Y et al (2017) Wind power forecasting errors modelling approach considering temporal and spatial dependence. J Mod Power Syst Clean Energy 5(3):489-498

[15] Liu H, Tian H, Li Y (2012) Comparison of two new ARIMAANN and ARIMA-Kalman hybrid methods for wind speed prediction. Appl Energy 98:415-424

[16] Amrouche B, Pivert X (2014) Artificial neural network based daily local forecasting for global solar radiation. Appl Energy 130:333-341

[17] Moazzami M, Khodabakhshian A, Hooshmand R (2013) A new hybrid day-ahead peak load forecasting method for Irans national grid. Appl Energy 101:489-501

[18] Torr H (2009) Electricity futures prices: some evidence on forecast power at Nord pool. J Energy Mark 2(3):3-25

[19] Yanga Z, Cea L, Liana L (2017) Electricity price forecasting by a hybrid model, combining wavelet transform, ARMA and kernel-based extreme learning machine methods. Appl Energy 190:291-305 
[20] Munkhammara J, Widna J, Rydnb J (2015) On a probability distribution model combining household power consumption, electric vehicle home-charging and photovoltaic power production. Appl Energy 142:135-143

[21] Montgomery D, Jennings C, Kulahchi M (2014) Introduction to time series analysis and forecasting-III. Wiley, New Jersey

[22] UN Faq (2015) Verification of the gross mass of packed containers by sea. https://www.gov.uk/government/publications/. Accessed 10 June 2015

[23] International Maritime Organization (2017) SOLAS container mass verification requirements. http://www.imo.org/en/ MediaCentre/HotTopics/container. Accessed 3 Apr 2017

[24] Papaioannou G, Dikaiakos C, Dramountanis A et al (2016) Analysis and modeling for short- to medium-term load forecasting using a hybrid manifold learning principal component model and comparison with classical statistical models (SARIMAX, exponential smoothing) and artificial intelligence models (ANN, SVM): the case of Greek electricity market. Energies. https://doi.org/10.3390/en9080635

Feras ALASALI received the M.Sc. degree in electrical power engineering at the Al-Yarmouk University. After graduation, he worked in Electrical Distribution Company as metering and protection engineer and then as MV/HV substation engineer. He is currently a Ph.D. student at the University of Reading working on control strategies for energy storage systems based on load forecasting.

Stephen HABEN is a postdoctoral research assistant at the University of Oxford and an academic partner on the New Thames Valley Vision Project as part of Ofgems Low Carbon Network Fund (LCNF). His main interests include forecasting, clustering methods, data assimilation, large data analytics, linear algebra and optimization.

Victor BECERRA received his B.Eng. degree in electrical engineering at Simon Bolivar University, Venezuela, in 1990, and his $\mathrm{Ph} . \mathrm{D}$. degree in control engineering from City University, London, in
1994. He is currently a professor of power systems engineering and deputy head at the School of Engineering, University of Portsmouth, UK. Between 1989 and 1991, he was employed as an electrical engineer at C.V.G. Edelca, Caracas, Venezuela. Between 1994 and 1999 he was a post-doctoral research fellow the Control Engineering Research Centre at City University, London. During the period between 2000 and 2015 he was an academic at the School of Systems Engineering, University of Reading, UK. During his career, he has received research funding from the EPSRC, the Royal Academy of Engineering, the European Space Agency, the Technology Strategy Board, the EU, and UK industry. His current research interests consider a range of issues related to electrical power systems, as well as the methods and applications of automatic control. These interests include control of power systems, energy storage and its integration to power grids, computational intelligence for smart grids, computational optimal control, nonlinear control, state estimation and robotics.

William HOLDERBAUM received the Ph.D. degree in automatic control from the University of Lille, Lille, France, in 1999. He was a research assistant with the University of Glasgow, Glasgow, UK, from 1999 to 2001 and the University of Reading as lecturer, senior lecturer and professor. He is currently a professor with the School of Engineering, Metropolitan Manchester University, Manchester, UK. His current research interests include control theory and its applications to control of energy storage. 